\title{
Universal Relativity: Absolute Time and Mass
}

\author{
Bandula Dahanayake \\ Farmfield Crescent, Kanata, Ontario, Canada
}

Email address:

Bandula_Dahanayake@yahoo.com

\section{To cite this article:}

Bandula Dahanayake. Universal Relativity: Absolute Time and Mass. International Journal of Astrophysics and Space Science. Vol. 3, No. 1, 2015, pp. 7-15. doi: 10.11648/j.jjass.20150301.12

\begin{abstract}
The nature of the universe is such the speed of light in a homogeneous medium is a constant that is determined by the permittivity and permeability of the medium including a vacuum; a path of light appears to depend on the observer's frame of reference. This led to believe that the time depends on the observer's frame of reference, which is an inherent assumption in the Special Relativity. The Special Relativity further stipulates that the mass depends on the observer's frame of reference. The both time dilation and mass dilation of moving objects are the foundation of the Special Relativity by human design; they are not inherent characteristics of the universe. In the Universal Theory of Relativity the time and mass are absolute. When an object is in motion, time and mass remain unchanged; however the geometry of the object contracts in all directions leading to the volume contraction. Since mass is absolute, the mass-density dilates due to the volume contraction. When an object reaches the speed of light, its mass-density reaches infinity while the mass remains unchanged; this gives rise to extreme close proximity gravitation within the boundary of the object at rest, which is the event horizon. In the Special Relativity, the contraction of the volume of a moving object is not taken into account, and hence the increase in gravity of a moving object is erroneously attributed to mass dilation, which led to the celebrated mass-energy relationship that does not hold when the time and mass are absolute. When time and mass are absolute what prevails is the mass-density and energy-density relationship. When an object is in motion, its gravity dilates as a result of volume contraction while the time and mass remain the same. In the Special Relativity, the time, mass, and length, all depend on the observer's frame of reference, and hence the corrections have to be made to all. However, in the Universal Relativity, only the change in the geometry has to be taken into account. An object moving in space at an acceleration $\mathrm{g}$, and an object at rest under gravitation $\mathrm{g}$ are not equivalent; the principle of equivalence does not hold. The gravity does not bend light, only a density gradient in a medium does. A second is always a second, and mass remains the same irrespective of the speed and the location of the object in the universe.
\end{abstract}

Keywords: Relativity, Time, Mass, Black-Holes, Gravity, Einstein, Time-Dilation, Special-Relativity, Equivalence

\section{Introduction}

With the introduction of the unified theory of Electromagnetic propagation in the late $1800^{\mathrm{s}}$, it became clear that the speed of light is a constant in a homogeneous medium. In the case of moving objects, relative velocities can be handled by using vector addition; the same could not be done for light due to its constant nature of speed that is determined by the medium including a vacuum. Though the speed of light is independent of the observer's frame of reference, the path light travels appears to depend on the observer's frame of reference. So the time taken for light to travel from one point to another appears to be different for different observers in different frames of reference. This gives the impression that the time is not universal, or not absolute. The notion that the time is different for different observer's frame of reference led to the development of the Special Relativity in 1905. The time and mass dilation were the foundation of the Special Relativity $[1,2,3,4]$.

In the Special Relativity, it was also conjectured, without any forward proof, that the mass of an object is dependent on the observer's frame of reference. There is no intuitive reason or justification to why mass should depend on the observer's frame of reference, or why should mass be relative; it is an unsubstantiated assumption.

If the path of light depends on observer's frame of reference, it is not because actual path the light traveled has changed. Once the light is out of the source, the actual path light takes is always determined by the medium, the space; nothing to do with the observer. The path, light travels, appears to depend on the observer's frame of reference because the observer is unaware of the changes in the geometry of the moving object 
that has taken place due to its motion. The path of light appeared to be altered with respect to the observer, even though in actual fact, the path of light relative to the medium always remains the same irrespective of the observer's frame of reference. In the case of a moving object, the actual path of light does not change; a time interval does not change; mass does not change; the only thing that changes is the geometry of the moving object.

The time and mass dilation of a moving object are not universal facts that we have to live by. It is a concept that was imposed on nature by human. We are going to restore the universal nature of the time and mass. We are going maintain the natural fact that the time and mass are absolute in the universe. It doesn't matter where in the universe you are or what speed you are travelling at, your clock runs just like everybody else's clock, no difference. Your mass at any speed will also be the same as your mass at rest. This is achieved simply by allowing a moving object to contract its geometry in all directions as it happens in the nature, and in effect giving the object the freedom to alter its volume.

The Special Relativity suffers from time dilation because it did not allow any lateral dimensional changes, changes in the cross section area perpendicular the direction of motion. The special Relativity severely restricted any lateral changes.

\section{Universal Facts}

\section{Axiom-1: Speed of Light}

The speed of light in a homogeneous medium is a constant that is determined solely by the permittivity and the permeability of the medium.

\section{Axiom-2: Path of Light}

Once the light is out of a source, the path of light is determined solely by the density gradient of the medium (or lack of it), nothing else.

\section{Axiom-3: Time}

Time is absolute. It does not depend on the observer's frame of reference. A second is a second everywhere in the universe.

\section{Axiom-4: Mass}

Mass is absolute. It does not depend on the observer's frame of reference. An object has the same mass everywhere in the universe, whether the object is at rest or moving.

\section{Axiom-5: Geometry}

Geometry is NOT absolute. The geometry of an object depends on the observer's frame of reference. The geometry contracts in all directions when an object is moving; the volume of a moving object is smaller than the volume of the same object at rest.

\section{Universal Relativity}

Let us assume the state of a moving object $\mathrm{O}$, with an observer inside, on frame $\mathrm{S}$ is, $\mathrm{O}[\mathrm{m}, \Delta \mathrm{t}, \ell, \mathrm{A}, \mathrm{V}, \mathrm{u}]$, where

$\mathrm{m}=$ mass of the moving object,

$\Delta \mathrm{t}=\mathrm{a}$ time interval on frame $\mathrm{S}$,

$\ell=$ length of the moving object in the direction of motion $\mathrm{u}$,

$\mathrm{A}=$ area of a cross section perpendicular to the velocity $\mathrm{u}$,

$\mathrm{V}=$ volume of the moving object,

$\mathrm{u}=$ speed of the object,

Then, the state of the object at rest $\mathrm{O}_{o}$ on a still frame of reference, $\mathrm{S}_{\mathrm{o}}$ can be obtained by substituting $\mathrm{u}=0$, i.e.,

$\mathrm{O}_{\mathrm{o}}\left[\mathrm{m}_{\mathrm{o}}, \Delta \mathrm{t}_{\mathrm{o}}, \ell_{\mathrm{o}}, \mathrm{A}_{\mathrm{o}}, \mathrm{V}_{\mathrm{o}}, \mathrm{u}=0\right]$, where

$\mathrm{m}_{\mathrm{o}}=$ mass of the object at rest,

$\Delta \mathrm{t}_{\mathrm{o}}=$ the time interval at rest on frame $\mathrm{S}_{\mathrm{o}}$,

$\ell_{\mathrm{o}}=$ length of the object at rest in the direction of $\mathrm{u}$,

$\mathrm{A}_{\mathrm{o}}=$ area of a cross section perpendicular to the direction $\mathrm{u}$, $\mathrm{V}_{\mathrm{o}}=$ volume of the object at rest,

According to the Axiom-3 and Axiom-4, the time and mass are absolute, and hence

$\mathrm{m}=\mathrm{m}_{\mathrm{o}}$

$\Delta \mathrm{t}=\Delta \mathrm{t}_{\mathrm{0}}$

Now, let us define the Universal Relativity Parameter $\alpha(u, c)=\left(1-u^{2} / c^{2}\right)^{1 / 2}$, where $\mathrm{c}$ is the speed of light in a homogeneous medium.

\subsection{The Lateral Dimension Contraction Theorem}

When the time is absolute, the lateral dimension $r$ on a cross section perpendicular to the direction of motion $u$ is given by, $\mathrm{r}=\alpha(\mathrm{u}, \mathrm{c}) \mathrm{r}_{\mathrm{o}}$.

The proof is given in the Appendix-1.

\subsection{The Area Contraction Theorem}

When time is absolute, the area A of a cross section of a moving object perpendicular to the direction of motion $u$ is given by, $\mathrm{A}=\alpha^{2}(\mathrm{u}, \mathrm{c}) \mathrm{A}_{\mathrm{o}}$.

The proof is given in the Appendix-1.

\subsection{The Length Contraction Theorem}

When time is absolute, the length $\ell$ of a moving object in the direction of the speed of motion $\mathrm{u}$ is given by, $\ell=\alpha^{2}(\mathrm{u}, \mathrm{c}) \ell_{\mathrm{o}}$. The proof is given in the Appendix-2.

\subsection{The Volume Contraction Theorem}

When the time is absolute, the volume $\mathrm{V}$ of a moving object is given by, $\mathrm{V}=\alpha^{4}(\mathrm{u}, \mathrm{c}) \mathrm{V}_{\mathrm{o}}$.

The proof is given in the Appendix-3.

\subsection{The Gravity of a Moving Object}

Lemma: The gravity of a moving object increases while the mass remains unchanged.

Proof: Consider a spherical object of radius $r_{o}$ at rest. Though each point on the surface of the sphere has equal distance from the center $\mathrm{O}$ when it is at rest, the moving object does not have an equal distance from the center to each point on the surface since the contraction along the direction of the 
motion, and the lateral contraction perpendicular to the direction of motion are different. Consider a point $\mathrm{P}$ on the surface at distance $r$ from the center of the moving object. The gravity at $\mathrm{P}$ will be a minimum when $\mathrm{OP}$ is perpendicular to the direction of motion. The center and the center of the gravity of the sphere are the same from symmetry.

The gravity $g$ of the moving object at point $\mathrm{P}$, as well as the gravity $\mathrm{g}_{\mathrm{o}}$ when the object is at rest, are given by,

$$
\begin{gathered}
\mathrm{g}=\mathrm{Gm} / \mathrm{r}^{2} \\
\mathrm{~g}_{\mathrm{o}}=\mathrm{Gm}_{\mathrm{o}} / \mathrm{r}_{\mathrm{o}}{ }^{2},
\end{gathered}
$$

where $\mathrm{G}$ is the gravitational constant, $\mathrm{m}=$ mass of the object, $\mathrm{r}=$ distance to point $\mathrm{P}$ on the surface from the center of gravity when the object is moving, $r_{0}=$ distance to point $P$ on the surface from the center of gravity when the object is at rest; from the Lateral Dimension Contraction Theorem $r \leq r_{0}$, i.e.

$$
\mathrm{g} / \mathrm{g}_{\mathrm{o}}=\left[\mathrm{m} / \mathrm{m}_{\mathrm{o}}\right]\left[\mathrm{r}_{\mathrm{o}}{ }^{2} / \mathrm{r}^{2}\right]
$$

Since mass is absolute, $\mathrm{m}=\mathrm{m}_{\mathrm{o}}$, we get

$$
\mathrm{g} / \mathrm{g}_{\mathrm{o}}=\mathrm{r}_{\mathrm{o}}{ }^{2} / \mathrm{r}^{2}
$$

Substituting for $r$ from the Lateral Dimension Contraction Theorem given in Section-3.1, we get,

$$
\begin{array}{r}
\mathrm{g} / \mathrm{g}_{\mathrm{o}}=\mathrm{r}_{\mathrm{o}}{ }^{2} /\left[\alpha(\mathrm{u}, \mathrm{c}) \mathrm{r}_{\mathrm{o}}\right]^{2}, \\
\text { i.e. } \mathrm{g}=\mathrm{g}_{\mathrm{o}} / \alpha^{2}(\mathrm{u}, \mathrm{c}) \\
\text { Since, } \alpha(\mathrm{u}, \mathrm{c})=\left(1-\mathrm{u}^{2} / \mathrm{c}^{2}\right)^{1 / 2} \text {, we get }
\end{array}
$$

$$
\mathrm{g}=\mathrm{g}_{\mathrm{o}} /\left(1-\mathrm{u}^{2} / \mathrm{c}^{2}\right)
$$

This indicates that the gravity of a moving object increases as the speed increases. As $\mathrm{u} \rightarrow \mathrm{c}, \mathrm{g} \rightarrow \infty$, i.e. when the speed of the object approaches the speed of light, the gravity of the object reaches infinity while the mass remains unchanged.

When $\mathrm{u}<<\mathrm{c}$, we get

$$
\begin{gathered}
\mathrm{gc}^{2}=\mathrm{g}_{\mathrm{o}} \mathrm{c}^{2}+\mathrm{g}_{\mathrm{o}} \mathrm{u}^{2}, \\
\text { or } \Delta \mathrm{gc}^{2}=\mathrm{g}_{\mathrm{o}} \mathrm{u}^{2}, \text { where } \Delta \mathrm{g}=\mathrm{g}-\mathrm{g}_{\mathrm{o}} .
\end{gathered}
$$

Substituting for $\mathrm{g}_{\mathrm{o}}$, we get,

$$
\Delta \mathrm{g}=\eta \mathrm{e},
$$

where $\mathrm{e}=(1 / 2) \mathrm{mu}^{2}$, the kinetic energy of the moving object, $\eta=2 \mathrm{G} /\left(\mathrm{r}_{\mathrm{o}}{ }^{2} \mathrm{c}^{2}\right)$, a constant.

It is the increase in gravity that the kinetic energy of an object is proportional to, not to the mass. By increasing the kinetic energy, we can increase the gravity of an object. Similarly, when the speed of an object deceases, the gravity decreases. The mass cannot be increased or decreased by changing its kinetic energy; mass is absolute.

In this relationship, the proportionality constant $\eta$ varies according to the point $\mathrm{P}$ chosen on the surface. If we chose a different point on the surface of the moving object, the distance to the center of gravity will be different and hence the proportionality constant $\eta$ will be different. The gravity $g$ is the maximum when, OP, the line connecting the center of gravity and $\mathrm{P}$ is on the direction of motion. It can be shown that the maximum change of gravity is given by

$$
\Delta \mathrm{g}_{\max }=\eta_{\max } \mathrm{e}
$$

where $\eta_{\max }=4 \mathrm{G} /\left(\mathrm{r}_{\mathrm{o}}{ }^{2} \mathrm{c}^{2}\right)$.

The parameter $\eta$ lies within the range given by,

$$
2 \mathrm{G} /\left(\mathrm{r}_{\mathrm{o}}{ }^{2} \mathrm{c}^{2}\right) \leq \eta \leq 4 \mathrm{G} /\left(\mathrm{r}_{\mathrm{o}}{ }^{2} \mathrm{c}^{2}\right) \text {. }
$$

When an object is moving, its mass remains constant, however its gravity increases due to the volume contraction. In the Special Relativity, this increase in gravity has been erroneously attributed to a mass dilation of a moving object that led to the relationship $\mathrm{e}=\mathrm{mc}^{2}$, which does not hold in reality when the time and mass are absolute. It is the gravity of a moving object that dilates, not the mass.

\section{The Relationship between Mass-Density an Energy-Density}

According to the Axiom-4, the mass is absolute. The mass does not depend on the observer's frame of reference. Since the mass is universal, it is not possible to change a mass of an object by changing the speed of the object. However, when an object is moving, volume of the object contracts and as a result the mass-density dilates.

\subsection{The Mass-Density Dilation Theorem}

When the time is absolute, the mass-density $\rho$ of a moving object is related to its mass-density $\rho_{o}$ at rest by the relationship $\rho=\rho_{\mathrm{o}} / \alpha^{4}(\mathrm{u}, \mathrm{c})$.

Proof: From the Volume Contraction Theorem, we have,

$$
\mathrm{V}=\alpha^{4}(\mathrm{u}, \mathrm{c}) \mathrm{V}_{\mathrm{o}}
$$

The mass density $\rho$ of a moving object is given by,

$$
\begin{gathered}
\rho=\mathrm{m} / \mathrm{V} \\
\rho=\mathrm{m} / \alpha^{4}(\mathrm{u}, \mathrm{c}) \mathrm{V}_{\mathrm{o}}
\end{gathered}
$$

Since $\mathrm{m}=\mathrm{m}_{\mathrm{o}}$ and $\rho_{\mathrm{o}}=\mathrm{m}_{\mathrm{o}} / \mathrm{V}_{\mathrm{o}}$

$$
\rho=\rho_{\mathrm{o}} / \alpha^{4}(u, c) \text {. }
$$

Corollary:

Only an object that has no mass can reach the speed of light. Proof: Let's consider the mass-density relationship,

$$
\rho=\rho_{\mathrm{o}} / \alpha^{4}(\mathrm{u}, \mathrm{c}) .
$$

Substituting for $\alpha(\mathrm{u}, \mathrm{c})$, we get,

$$
\rho=\rho_{\mathrm{o}} /\left(1-\mathrm{u}^{2} / \mathrm{c}^{2}\right)^{2}
$$

When $\mathrm{u}=\mathrm{c}, \rho$ is undefined. However, as $\mathrm{u} \rightarrow \mathrm{c}$, if $\mathrm{m} \rightarrow 0$, then, the equality holds. Hence, only an object of zero mass can reach the velocity of light in a given medium. This agrees with the nature anywhere in the universe since the photons, the light itself which is mass-less, travels at speed c.

\subsection{Mass-Density and Kinetic-Energy-Density Equivalence}

Let us consider the mass-density relationship,

$$
\rho=\rho_{o}\left(1-u^{2} / c^{2}\right)^{-2}
$$


When $\mathrm{u}<<\mathrm{c}, \rho$ can be approximated to,

$$
\rho=\rho_{o}\left(1+2 u^{2} / c^{2}\right),
$$

i.e. $\Delta \rho c^{2}=2 \rho_{o} u^{2}$, where $\Delta \rho=\rho-\rho_{0}$.

As expected the mass-density and kinetic-energy has the exactly the same form as the gravity and the kinetic-energy relationship; this is because the increase in gravity is the result of the increase in mass-density. This equivalence indicates how mass-density is translated into kinetic-energy-density and vice versa. By increasing the speed of an object, we can increase the mass-density of an object and vice versa. If the mass-density of an object decreases, that results in a slowing down of the object. The mass always remains the same. It is not possible to increase the mass of an object by increasing its speed; the mass of an object cannot be reduced by decreasing the speed of the object either.

Definition: Mass

The mass is a measure of the substance in an object, not the energy.

Contrary to popular belief, the energy and the mass are not one and the same. The energy does not have a mass. A mass can be converted to energy and vice versa, if you have a mechanism to do it. The nature does this transformation in living cells by using chlorophylls in plants and mitochondria in other living cells; weak electromagnetic force (radiation) in atoms and simple fire do this too. The energy does not have a mass until the energy is converted into mass by some means; similarly, the mass does not have energy until the mass is converted into energy by some means. Mass and energy are two separate entities that can be converted to one another by some mechanism. People have been working out to lose mass since the dawn of the civilization. Given mass $m$, the total mass $\mathrm{m}$ cannot be converted to energy e, and similarly, given energy e, the total energy e cannot be converted to a mass m; therefore mass and energy cannot be used interchangeably.

\subsection{Transient-Black Holes}

The mass-density $\rho$ of a moving object is given by,

$$
\rho=\rho_{0}\left(1-u^{2} / c^{2}\right)^{-2} .
$$

When $\mathrm{u} \rightarrow \mathrm{c}, \rho \rightarrow \infty$. As u approaches $\mathrm{c}$, the mass-density of the object approaches infinity creating a massive gravitational field around it in the close proximity. In other words, when an object that has a mass reaches a speed closer to the speed of light, the object becomes a transient-black-hole.

Definition: Transient-Black-Hole:

An object that reaches a speed close to the speed of light is defined as a transient-black-hole. As speed slows down, a transient-black-hole turns back to an ordinary object.

The transient-black-holes are different from the conventional black-holes that are formed in the process of collapsing stars. Transient-black-holes can turns back to an ordinary object when the speed of the object slows down. The mass of a transient-black-hole is the same as the mass of the object at rest. As the object reaches the speed of light, the volume approaches zero, driving mass-density infinitely large.
Since the volume of the object is now close to zero, the entropy of the object, which is now a transient-black-hole, is nearly zero. So, as the speed of an object reaches the speed of light, the entropy of the object approaches zero. Therefore, transient-black-holes contain nearly zero entropy.

If the object of volume $V_{o}$ is a sphere of radius $r_{o}$, then, gravity remains normal outside $r_{0}$. When the object reaches a speed close to the speed of light, the volume contracts and reaches almost zero. The factor $1 / \mathrm{r}^{2}$, that the gravity is proportional to, becomes extremely large when $\mathrm{r} \rightarrow 0$; this is what create the extreme gravity within radius $r_{o}$ or within the boundaries of volume $\mathrm{V}_{\mathrm{o}}$. Any object comes closer than distance $r_{o}$ will be subjected to this gravitational force. Since all the extreme events are within the radius $r_{o}$, the radius $r_{o}$ or the boundary of volume $\mathrm{V}_{\mathrm{o}}$ becomes the event horizon of the transient-black-hole.

Since the object that approaches the velocity of light has a massive gravitation at close proximity, it absorbs any mass that happens to come by within $r_{0}$. As it attracts nearby masses, its speed slows down. The new speed $\mathrm{u}_{\text {new }}$ after absorbing a new mass, $\mathrm{m}_{\mathrm{f}}$ moving in the same direction at speed $\mathrm{u}_{\mathrm{f}}$ is given by,

$$
\mathrm{u}_{\text {new }}=\left(\mathrm{mu}+\mathrm{m}_{\mathrm{f}} \mathrm{u}_{\mathrm{f}}\right) /\left(\mathrm{m}+\mathrm{m}_{\mathrm{f}}\right)
$$

where, $\mathrm{m}$ is the mass of the transient-black-hole, or mass of the object that turns into a transient-black-hole. When new mass is fallen to a transient-black-hole, the speed of the transient-black-hole slows down as given in eqn. (1). As speed slows down, the volume of the moving object increases, in effect, increasing the entropy. When the volume of a transient-black-hole increases, its mass-density decreases; this, in effect, reduces the gravitational field and transforms the transient-black-hole back into a normal object at a speed well below the speed of light. So, a transient-black-hole eventually will transform back into a normal object. However, the mass of the new object is $\mathrm{m}+\mathrm{m}_{\mathrm{f}}$. Since mass is absolute, Newton's laws applies at anytime anywhere in the universe including transient-black-holes.

The idea that a mass fallen into a black-hole gets stretched and turns into spaghetti like shape may not be true because gravitational action is so fast there isn't enough time for the change in shape to take place.

\subsection{Why Black-Holes are Black}

The reason black-holes are black is not their massive gravity, rather its massive density. The gravity cannot exert a force on mass-less object like light, hence it is not the gravity acting directly on light that prevents light coming out of a black-holes; it is rather the medium density inside the black-hole that prevents light coming out of black-holes, making black-holes black. The medium density inside a black-hole is such no oncoming light is reflected back (total absorption), and there is no outgoing light either (total internal reflection); it is the case when a density of a medium is very high, as in the case of black-holes, just like in the deep ocean. 


\section{Gravity and Electromagnetic Waves}

\subsection{The Effect of Gravity on Light}

Property: Only a density gradient in a medium can bend light, nothing else.

The gravity exerts a force only on objects that has a mass. Since the light has no mass, the gravity has no direct influence on light. However, gravity can exert an indirect influence on light through the medium; near the earth the medium is air. The velocity of light is same everywhere in the medium if the medium is homogeneous. A gravitational object disturbs the homogeneity of the medium creating a density gradient in the medium. The density is higher closer to the gravitational objects and decreases with the distance. This density gradient in the medium creates permittivity gradient and permeability gradient in the medium. Closer it is to the gravitational object, the higher are the permittivity $(\varepsilon)$ and permeability $(\mu)$, and they decrease with the distance from the gravitational object. Since the velocity of light $c=1 /(\varepsilon \mu)^{1 / 2}[5]$, the closer the light reaches the gravitational object, the slower the speed of light will be. This slowing of velocity of light creates refraction exactly similar to the fiber optic cables. This refraction of light closer to a gravitational object, or gravitational-lensing, is an indirect influence of gravity on light through the medium. The gravity cannot exert any direct influence on any object that is mass-less including light. If the medium is a perfect vacuum, then, there will not be any refraction of light near a gravitational object. However, there are no perfect vacuums. So, there will always be some refraction of light near a gravitational object due to permittivity and permeability gradient present in the vicinity of gravitational object. Some refraction of light near the earth is always there due to the density gradient of air near the earth; this refraction is not significant since the earth's gravitation is weak.

Lemma-1:

The gravity has no effect on light in a vacuum.

Proof: Consider a gravitational object in a vacuum. Assume a beam of light travelling directly toward the gravitational object. If gravity has an effect on the light, the light beam has to accelerate under gravity causing its speed to increase as it approaches the gravitational object; that is what gravity does to objects, it accelerates any mass. The gravity attracts other masses, whatever the object that is. This is a contradiction since the speed of light in a vacuum is a constant. Therefore, gravity has no effect on light in a vacuum. If light has a mass, its velocity will not be a constant under gravity; though light has energy, it does not have a mass. This does not allow us to consider energy as mass equivalent; this leads to the following lemma.

Lemma-2:

If energy and mass are equivalent, velocity of light will not be a constant under gravity in a vacuum.

Lemma-3:

Gravity does not induce a frequency shift in light in a vacuum.

Proof: An increase in energy of an object moving toward a gravitational object is due to the increase of the velocity of the object due to gravitational acceleration. So without increase in speed of an object, there would be no increase in energy of the object under gravity. Since the gravity can't increase the speed of light, there would be no increase of energy that leads to a frequency shift in the light beam either. So, gravity does not create a frequency shift of light in a vacuum. The gravity can have an indirect influence on the frequency spectrum of light in the presence of a medium.

\subsection{Gravity and Frequency Shift of Light Spectrum in the Presence of a Medium}

Consider a point $\mathrm{P}$ at distance $\mathrm{r}$ from a gravitational object. Let the speed of light at point $\mathrm{P}$ be $\mathrm{c}(\mathrm{r})$, which is a function of distance $r$ as we have seen in previous section. Now, at point $P$, for any given frequency $f$, we have the wavelength $\lambda(r)$ given by,

$$
c(r)=f \lambda(r) .
$$

When $\mathrm{r} \rightarrow \infty$, we have a point far away from the gravitational object where we can assume the medium is homogeneous, so, we have $\mathrm{c}(\mathrm{r} \rightarrow \infty)=\mathrm{c}$, and $\lambda(\mathrm{r} \rightarrow \infty)=\lambda$. So, a point far away from gravitational object, the medium is homogeneous and hence the wavelength $\lambda$ for the same frequency $\mathrm{f}$ is given by,

$$
\mathrm{c}=\mathrm{f} \lambda \text {. }
$$

Dividing eqn. (2) by eqn. (3), we get, $\mathrm{c}(\mathrm{r}) / \mathrm{c}=\lambda(\mathrm{r}) / \lambda$, i.e. $\lambda(r)=[c(r) / c] \lambda$

Since $\mathrm{c}(\mathrm{r})<\mathrm{c}$, we get, $\lambda(\mathrm{r})<\lambda$. As light reaches a gravitational object, wavelength of light shortens due to the density gradient of the medium. In other words, a gravitational object alters the homogeneity of the medium, in effect indirectly creating a blue-shift on approaching light spectrum. When light travel from high density medium to low density medium, the effect will be reversed causing a red shift. There will be no blue-shift or red-shift in a perfect vacuum.

When light enters a positive density gradient in the medium, the light slows down and the change of energy will be stored as a blue shift in the spectrum. As the refracted light leaves the density gradient, the energy is retrieved from the frequency spectrum to restore the speed of light, in effect creating a red-shift in the spectrum. The blue-shift created when light enters a medium density gradient cancels out with the red-shift created when the light is leaving the density gradient in the medium, in effect, there is no net energy gain or loss; this leads to the following lemma.

Lemma-1:

Positive density gradient in a medium creates a blue-shift in the light spectrum while a negative density gradient of the medium creates a red-shift.

Lemma-2

Change of velocity of light due to change in medium density leads to a frequency shift in the light spectrum.

\subsection{Fallacy of Principle of Equivalence on Gravity}

The Principle of Equivalence on gravity $[1,6,7]$ is 
fundamentally inaccurate. The Principle of Equivalence states that, "an object at rest on a gravitational object under gravity $g$ is equivalent to an object moving at acceleration $\mathrm{g}$ in space; no observer inside a sealed cabin can distinguish if the cabin is at rest on a gravitational object under gravity $g$, or if the cabin is moving at acceleration $g$ in space".

Let's consider a sealed cabin with an observer inside. Inside the cabin is a vacuum so there will not be any medium density gradient in the presence of gravity leading to a velocity of light gradient which make light to bend. Relative to a medium, only thing that can bend light is a density gradient in a medium. Since inside the cabin is a vacuum, its homogeneity is not affected by the presence of gravity and hence the speed and the path of light remains unaltered.

Now consider a sealed cabin with an observer inside at rest on a gravitational object under gravity g. Assume a light beam is fired horizontally inside the cabin. Since the cabin is not moving, observer sees a horizontally straight light beam; the gravity has no effect on mass-less objects in a vacuum; the light energy has no mass.

Now, let us consider that the cabin is moving at an acceleration $\mathrm{g}$ in space. Assume the same light beam perpendicular to the direction of motion inside the cabin. The medium inside the cabin is a vacuum. The cabin is moving up in space at an acceleration $\mathrm{g}$. With respect the medium, the light beam is moving at constant speed c horizontally, while the observer, with respect to the medium, is moving upward at acceleration g. So, the observer sees that the light is taking a curved path downward.

The observer sees a horizontally straight light path in the case of the cabin at rest (not moving) on a gravitational object under gravity g. The same observer sees a light beam taking a curved path when the cabin is moving at an acceleration $\mathrm{g}$ in space. If the Principle of Equivalence holds, the observer must have seen the same scenario for both situations; the observer does not see the same scenario.

Instead of a light beam, if golf balls were thrown, the balls take curved paths in both cases and hence observer is unable to distinguish any difference between the cabin still on a gravitational object with gravity $\mathrm{g}$, and the one moving in space at acceleration $g$; because unlike the path and the speed of the light, a path and speed of a golf ball is not solely determined by the properties of the vacuum or the medium. Since a golf ball has a mass, it has nonzero inertia; a golf ball is part of the cabin, the ball follows the cabin. Contrary to a golf ball, light has no mass and hence no inertia; the light beam is not a part of the cabin hence the light does not follow the cabin when the light pulse is released from the source and travelling in the medium. By observing the path of a light beam, an observer can easily determine that the two situations are different; in one situation cabin is moving at acceleration $g$ in space, while in the other case cabin is at rest on the ground under $g$.

An observer in a sealed cabin in an inertial frame can also determine if the cabin is at rest or moving at a constant speed by using a light beam (Appendix-1); the observer is not able to distinguish the two situations throwing golf balls.
The gravity does not bend light; it is a density gradient in a medium that bends light. It is the medium that bends light. If gravity bends light it should have appeared in the Maxwell's equations. It is the change in the speed of light in the medium that bends light. The light will bend only if the medium density gradient is such there is a permittivity and permeability gradient creating velocity gradient of light. The gravity has no effect on light in a vacuum. The Principle of Equivalence on gravity does not hold.

\section{Universe is Not Expanding}

The observation that the galaxies are moving away at an accelerated rate radially led to hypothesize that the universe is expanding at an accelerated rate. The universe is not expanding [8]. Some galaxies are moving away due to the increase in mass, while others are moving inward due to the mass loss.

The radial velocity $\mathrm{v}(\mathrm{t})$ and the radial acceleration $\frac{d}{d t} \mathrm{v}(\mathrm{t})$ of galaxies are given by,

$$
\begin{gathered}
\mathrm{v}(\mathrm{t})=\mathrm{H}(\mathrm{t}) \mathrm{r}(\mathrm{t}), \frac{d}{d t} \mathrm{v}(\mathrm{t})=\gamma(\mathrm{t}) \mathrm{r}(\mathrm{t}), \text { where } \\
\mathrm{H}(\mathrm{t})=2 \frac{d}{d t} \ln \mathrm{M}(\mathrm{t}), \gamma(\mathrm{t})=\mathrm{H}^{2}(\mathrm{t})+\frac{d}{d t} \mathrm{H}(\mathrm{t})
\end{gathered}
$$

$\mathrm{M}(\mathrm{t})=$ the mass of the galaxy, $\mathrm{r}(\mathrm{t})=$ radial distance, and

$\mathrm{v}(\mathrm{t})=$ radial velocity, velocity at which galaxies are moving inward or outward, $\mathrm{H}(\mathrm{t})=$ orbit dilation parameter.

Larger the radial distance to a galaxy, faster is its radial speed and acceleration.

When the mass of a galaxy or a planet increases, it moves away radially (orbit dilation); on the other hand, when the mass of a galaxy or a planet decreases, it moves inwardly (orbit contraction). The decrease of the mass of earth makes the earth move toward the sun (orbit contraction) creating Global Warming.

\section{Conclusions}

The Special Relativity of Einstein suffers from time and mass dilation of moving objects by its assumptions and design; not because time and mass dilation are inherent characteristics of the universe. The Special Relativity makes the assumption that the time and the mass dilations are inherent characteristics of the universe and build the theory around it. The Special Relativity assumes that a cross section area of an object perpendicular to the direction of motion remains the same when the object is moving; this is what led to the time dilation. Time dilation in the Special Relativity can be removed by removing this restriction. The mass dilation of moving objects is an unnecessarily imposed assumption, and had been incorporated into the Special Relativity for no apparent reason, just by choice without a proper justification. The time and mass dilation are not universal facts.

The time and mass are absolute. A second is a second everywhere in the universe. A mass, $m$ of an object is always mass, $m$ anywhere in the universe irrespective of the speed of 
the object. The time and mass are independent of the observer's frame of reference. What is not absolute is the geometry and hence the volume of an object. The geometry of a moving object depends on the observer's frame of reference. The length along the direction of motion and the lateral dimensions contracts when an object is in motion. As a result, the volume of the object shrinks. The higher is the speed of the object, the smaller is its volume. An object contracts in all of its dimensions as it is moving; the length along the direction of motion contracts much more than the lateral dimensions.

When the volume contracts, the distance from the center of gravity to any point on the surface decreases; this in effect creates increased gravitational force on the surface. So when an object is in motion, its gravity dilates while mass remains unchanged. In the Special Relativity, this gravity dilation had been erroneously interpreted as a mass dilation; the celebrated $\mathrm{e}=\mathrm{mc}^{2}$ relationship is a result of this error.

Since the mass remains unchanged, as an object gains speed, its volume decreases, in effect resulting a mass density increase. When an object reaches speed close to velocity of light, its mass density will be infinitely high within close proximity that it, in effect, turns itself into a transient-black-hole. Transient-black-holes are not necessarily massive objects. An object of any mass, even a grain of rice that reaches speed close to speed of light turns itself into a transient-black-hole. The extreme gravity is present only within the boundary of the object at rest, or within the event horizon; outside this boundary gravity remains normal.

The gravity is extreme near the gravitational center of a black-hole, an object that come close doesn't have sufficient time to change into a spaghetti-like shape along the way, and falls instantaneously. As transient-black-hole attracts other masses at close proximity, its speed slows down and regains the volume, and eventually turns itself into an ordinary object with ordinary gravity.

The black holes are black because of extremely high medium density, and nothing to do with gravity. The extremely high medium density gives rise to high permittivity and permeability resulting total internal reflection and total absorption of the light; incoming light is totally absorbed and outgoing light is totally reflected back inside creating total darkness. It doesn't matter how large the gravity is, the gravity doesn't attract light or any other mass-less objects. Since energy has no mass, the gravity does not prevent the electro-magnetic radiation taking place in a black-hole either. These are equally true for transient-black-holes as well as conventional black-holes that result from collapsing stars.

The change of mass-density of a moving object and its kinetic-energy-density are related by the equivalence, $\Delta \rho c^{2}=2 \rho_{\mathrm{o}} \mathrm{u}^{2}$. This relationship provides the energy-density and mass-density transfer equivalence. One can be transformed into other and vice versa, if you know how.

The mass and energy are not one and the same. A mass can be transformed into energy and vice versa, if you know how to do it. Energy does not have a mass until energy is transformed into a mass by some means; similarly, mass is not energy until mass is transformed into energy by some means. The nature does this dual transformation using chlorophyll and mitochondria in living cells, and using weak electromagnetic force (radiation) and fire in the rest. People have been working out to lose mass since the end of hunter gatherer civilization.

The mass and energy cannot be used interchangeably, because for a given a mass $\mathrm{m}$, the total mass $\mathrm{m}$ cannot be converted into energy e, and similarly, for a given energy e, the total energy e cannot be transformed into mass $\mathrm{m}$.

Newton's laws apply to all the moving bodies in the universe. The gravity has no effect on mass-less objects, and hence has no direct effect on the light. The refraction of light near a gravitational object is the result of homogeneity disturbance of the medium by the gravitational object, which in effect makes the velocity of light a function of distance from the gravitational object. This change of velocity with the distance creates a refraction of light exactly the same way as in fiber optic cables; a lensing effect. The changes in the speed of light due to the changes in the medium density created by a gravitational object also lead to a frequency shift of the light spectrum. The light travelling through increasing medium density gradient leads to a blue-shift of the spectrum; the light travelling through a decreasing medium density gives rise to a red-shift in the spectrum. Gravity can't bend light, only a medium can bend light. Gravity does not accelerate or decelerate light. Any influence of gravity on light is always through the medium density alteration. The gravity has no effect on light in a vacuum. Since the time and mass are absolute, $\mathrm{e} \neq \mathrm{mc}^{2}$; what holds is the equivalence $\Delta \rho \mathrm{c}^{2}=2 \rho_{\mathrm{o}} \mathrm{u}^{2}$.

The Principle of Equivalence on gravity, which states that an object at rest on a gravitational object under gravity $g$ is equivalent to an object moving in space at an acceleration $\mathrm{g}$, is not accurate. In fact, an observer in a sealed cabin can easily determine if the cabin is at rest on a gravitational object under gravity $g$ or if the cabin is moving at acceleration $g$ in space by using a light beam which behaves differently in two situations. In the case of a cabin at rest on a gravitational object under gravity g, an observer sees a straight light path, while the same observer sees a curved path in the case of moving (moving is the key) cabin in space at an acceleration g. Though the observer can't distinguish the two situations using golf balls, observer can certainly discriminate the two situations using a light beam. The Principle of Equivalence on gravity does not hold true.

An hour is an hour everywhere in the universe. A mass $m$ is also mass $m$ anywhere in the universe. Although the light has energy, the light does not have a mass and hence the gravity does not attract light. If you are planning a space flight to stay young, you are wasting your money and time; there is no twin paradox. The time passes equally everywhere in the universe.

In the Special Relativity, the time, mass, and the length are all relative. When dealing with moving objects using the Special Relativity, appropriate corrections have to be applied to the time, mass as well as to the length. However, if the Universal Relativity is used in handling moving objects, only the geometry of the object has to be corrected appropriately, all the rest remain the same. 


\section{Appendix-1}

The Proof of Area Contraction Theorem:

Without loss of generality, let's assume we have a cylindrical object with an observer sitting inside. The state of the moving cylinder $\mathrm{O}$ on frame $\mathrm{S}$,

$\mathrm{O}[\mathrm{m}, \Delta \mathrm{t}, \ell, \mathrm{A}, \mathrm{V}, \mathrm{u}]$, where,

$\mathrm{u}=$ the velocity of the object along the axis of the cylinder,

$\ell=$ the length of the cylinder along the axis,

$\mathrm{r}=$ the radius of the cylinder, $\mathrm{A}=\pi \mathrm{r}^{2}, \mathrm{~V}=\pi \mathrm{r}^{2} \ell$.

The state of the object at rest $\mathrm{O}_{0}$ in the frame $\mathrm{S}_{\mathrm{o}}$ is

$\mathrm{O}_{\mathrm{o}}\left[\mathrm{m}_{\mathrm{o}}, \Delta \mathrm{t}_{\mathrm{o}}, \ell_{\mathrm{o}}, \mathrm{A}_{\mathrm{o}}, \mathrm{V}_{\mathrm{o}}, \mathrm{u}=0\right]$, where $\mathrm{A}_{\mathrm{o}}=\pi \mathrm{r}_{\mathrm{o}}{ }^{2}, \mathrm{~V}_{\mathrm{o}}=\pi \mathrm{r}_{\mathrm{o}}{ }^{2} \ell_{\mathrm{o}}$,

$\mathrm{V}_{\mathrm{o}}=$ the volume of the cylinder at rest in the frame $\mathrm{S}_{\mathrm{o}}$,

$r_{0}=$ the radius of the cylinder at rest in the frame $S_{0}$,

$\ell_{\mathrm{o}}=$ length of the cylinder at rest in the frame $S_{0}$.

Now, let us consider a cross section of the cylinder that passes through the main axis. By rotating the cross section around the axis, we can obtain any cross section of the cylinder. Hence, by symmetry, any development for one cross section equally applies to any other cross sections since the object is moving along the main axis.

Consider, we have a light clock at $\mathrm{P}$ and a reflector at $\mathrm{Q}$. The light source is directed at $\mathrm{Q}$ as shown in Figure-1.1.

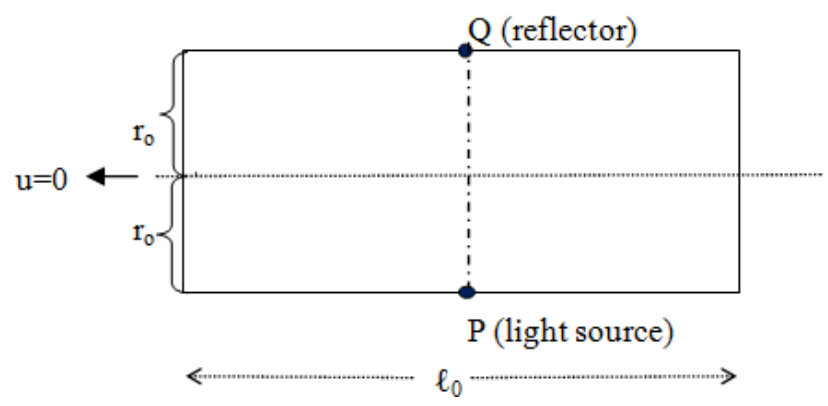

Figure-1.1. Object at Rest, Frame $S_{o}$

On Frame $\mathrm{S}_{\mathrm{o}}$, the time interval $\Delta \mathrm{t}_{\mathrm{o}}$ that takes light to travel from $\mathrm{P}$ to $\mathrm{Q}$ and $\mathrm{Q}$ to $\mathrm{P}$ is given by,

$$
\Delta \mathrm{t}_{\mathrm{o}}=2\left(2 \mathrm{r}_{\mathrm{o}} / \mathrm{c}\right)
$$

Now, let's consider the same object moving at speed $u$ along the axis as shown in Figure-1.2 on Frame S.

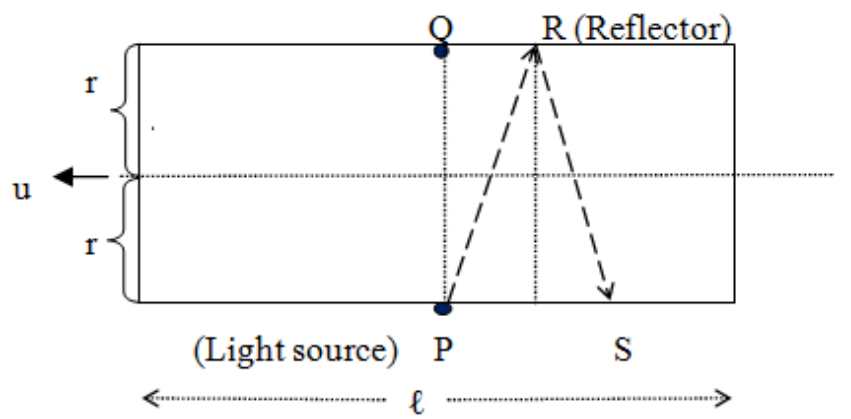

Figure-1.2. Moving Object, Frame S

Here, the observer sees the light path PRS. Let's consider time taken for light to travel from $\mathrm{P}$ to $\mathrm{R}$ and $\mathrm{R}$ to $\mathrm{S}$ is $\Delta \mathrm{t}$, then

$$
\Delta \mathrm{t}=(\mathrm{PR}+\mathrm{RS}) / \mathrm{c} .
$$

Since $\left.P R=R S, \Delta t / 2=P R / c, P R=\left[(u \Delta t / 2)^{2}+(2 r)^{2}\right)\right]^{1 / 2}$

$$
\Delta \mathrm{t}=2\left[2 \mathrm{r} /\left(\mathrm{c}^{2}-\mathrm{u}^{2}\right)^{1 / 2}\right] .
$$

Since time is absolute, $\Delta \mathrm{t}=\Delta \mathrm{t}_{\mathrm{o}}$

Substituting from eqns. (1.1) and (1.2), we get,

$$
\begin{gathered}
2\left[2 \mathrm{r} /\left(\mathrm{c}^{2}-\mathrm{u}^{2}\right)^{1 / 2}\right]=2\left(2 \mathrm{r}_{\mathrm{o}} / \mathrm{c}\right) \\
\mathrm{r}=\alpha(\mathrm{u}, \mathrm{c}) \mathrm{r}_{\mathrm{o}}
\end{gathered}
$$

where $\alpha(u, c)=\left(1-u^{2} / c^{2}\right)^{1 / 2}$.

The area, A of the moving object is given by, $A=\pi r^{2}$

Substituting for $r$ from eqn. (1.3), we get,

$$
\mathrm{A}=\pi\left[\alpha(\mathrm{u}, \mathrm{c}) \mathrm{r}_{\mathrm{o}}\right)^{2}
$$

Since $A_{o}=\pi r_{o}{ }^{2}$, we get $A=\alpha^{2}(u, c) A_{0}$

\section{Determining the State of an Inertial Frame:}

An observer sitting inside the cylinder can determine if the cylinder is at rest or moving by looking at the light path trajectory. If the light pulse takes a straight path $\mathrm{PQ}$, then the cylinder is still. On the other hand if the observer sees an angular path PR, then the cylinder is moving. Though the observer is unable to determine if the cylinder is at rest or moving by observing the path of a golf ball, the observer can determine the state of the cylinder by observing the trajectory of a light pulse. A golf ball has a mass and hence it has a momentum; this makes the golf ball a part of the object. Contrary to golf balls, light has no mass and hence no momentum; once the light has left the source, it is not a part of the object; the velocity of the light pulse is determined by the medium alone. This is the property that allows an observer to determine the state of the object using a light pulse.

\section{Appendix-2}

The Proof of Length Contraction Theorem:

Consider the same cylindrical object. This time we place the light source, $\mathrm{P}^{\prime}$ at the center of the left surface and the reflector, $\mathrm{Q}^{\prime}$ at the center of the right surface as shown in Figure-2.1.

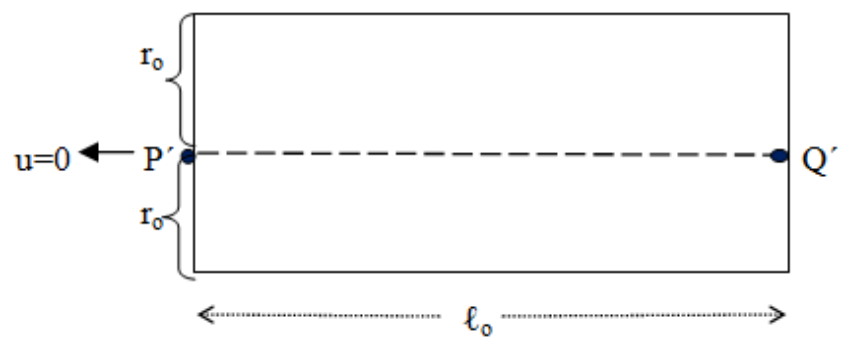

Figure-2.1. Object at Rest, Frame $S_{o}$

Let's consider that the time taken for light pulse to travel from $\mathrm{P}^{\prime}$ to $\mathrm{Q}^{\prime}$ and $\mathrm{Q}^{\prime}$ to $\mathrm{P}^{\prime}$ in frame $\mathrm{S}_{\mathrm{o}}$ is $\Delta \mathrm{t}_{\mathrm{o}}{ }^{\prime}$, then, we get,

$$
\Delta \mathrm{t}_{\mathrm{o}}{ }^{\prime}=2 \ell_{\mathrm{o}} / \mathrm{c}
$$


Now, consider the same object moving at speed $u$ along the axis to the left as shown in Figure-2.2.

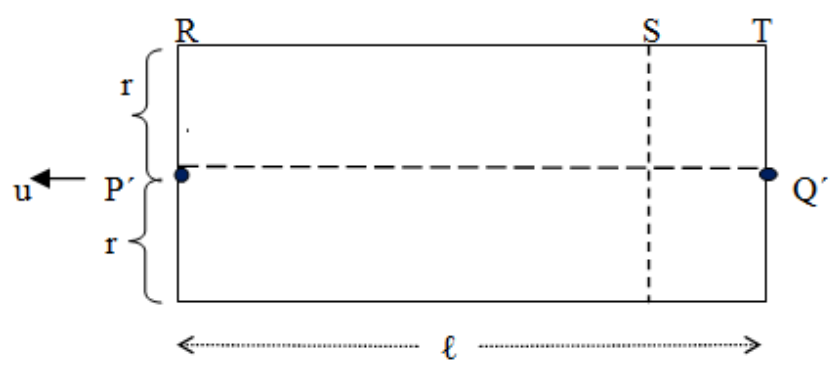

Figure-2.2. Moving Object, Frame S

Let us assume the time taken for light pulse to travel from $\mathrm{P}^{\prime}$ to $\mathrm{Q}^{\prime}$ on frame $\mathrm{S}$ is $\Delta \mathrm{t}_{\mathrm{f}}{ }^{\prime}$. Since the object is moving against the direction of the light pulse, the distance light pulse has to travel to reach the reflector is $\left(\ell-\mathrm{u} \Delta \mathrm{t}_{\mathrm{f}}{ }^{\prime}\right)$. Therefore, we get,

$$
\Delta \mathrm{t}_{\mathrm{f}}{ }^{\prime}=\left(\ell-\mathrm{u} \Delta \mathrm{t}_{\mathrm{f}}{ }^{\prime}\right) / \mathrm{c}
$$

Now, let us consider the backward travel time from $\mathrm{Q}^{\prime}$ to $\mathrm{P}^{\prime}$ is $\Delta \mathrm{t}_{\mathrm{b}}{ }^{\prime}$. This time, the object is moving at the same direction as the reflected pulse. When the reflected pulse reaches the source $\mathrm{P}^{\prime}$, the object has advanced by a distance of $\mathrm{u} \Delta \mathrm{t}_{\mathrm{b}}{ }^{\prime}$. So the reflected pulse has to travel $\left(\ell+\mathrm{u} \Delta \mathrm{t}_{\mathrm{b}}{ }^{\prime}\right)$ distance to reach the source $\mathrm{P}^{\prime}$.

The backward travel time from $\mathrm{Q}^{\prime}$ to $\mathrm{P}^{\prime}, \Delta \mathrm{t}_{\mathrm{b}}{ }^{\prime}$ is given by,

$$
\Delta \mathrm{t}_{\mathrm{b}}{ }^{\prime}=\left(\ell+\mathrm{u} \Delta \mathrm{t}_{\mathrm{b}}{ }^{\prime}\right) / \mathrm{c}
$$

The total travel time of the pulse from $\mathrm{P}^{\prime}$ to $\mathrm{Q}^{\prime}$ and $\mathrm{Q}^{\prime}$ to $\mathrm{P}^{\prime}$ is given by,

$$
\Delta \mathrm{t}^{\prime}=\Delta \mathrm{t}_{\mathrm{f}}{ }^{\prime}+\Delta \mathrm{t}_{\mathrm{b}}{ }^{\prime}
$$

Combining eqns. (2.2), (2.3), and (2.4), we get,

$$
\Delta \mathrm{t}^{\prime}=2 \ell \mathrm{c} /\left(\mathrm{c}^{2}-\mathrm{u}^{2}\right)
$$

If the time is absolute, the travel time $\Delta \mathrm{t}_{\mathrm{o}}{ }^{\prime}$ on frame $\mathrm{S}_{\mathrm{o}}$ must be the same as the travel time $\Delta \mathrm{t}^{\prime}$ on the moving frame $\mathrm{S}$.

Since time is absolute $\Delta \mathrm{t}_{\mathrm{o}}{ }^{\prime}=\Delta \mathrm{t}^{\prime}$.

Substituting from eqns. (2.1) and (2.5), we get,

$$
2 \ell_{\mathrm{o}} / \mathrm{c}=2 \ell \mathrm{c} /\left(\mathrm{c}^{2}-\mathrm{u}^{2}\right)
$$

$$
\ell=\alpha^{2}(\mathrm{u}, \mathrm{c}) \ell_{\mathrm{o}}
$$

where, $\alpha(u, c)=\left(1-u^{2} / c^{2}\right)^{1 / 2}$.

\section{Appendix-3}

The Proof of Volume Contraction Theorem:

From the area contraction theorem, we have,

$$
\mathrm{A}=\alpha^{2}(\mathrm{u}, \mathrm{c}) \mathrm{A}_{\mathrm{o}}
$$

From the length contraction theorem, we have,

$$
\ell=\alpha^{2}(\mathrm{u}, \mathrm{c}) \ell_{\mathrm{o}}
$$

The volume $\mathrm{V}$ of a moving cylindrical object is given by,

$$
\mathrm{V}=\mathrm{A} \ell
$$

Substituting from eqns. (3.1) and (3.2), we get,

$$
\mathrm{V}=\alpha^{4}(\mathrm{u}, \mathrm{c}) \mathrm{V}_{\mathrm{o}}
$$

where $V_{o}=A_{o} \ell_{0}$

\section{References}

[1] Einstein Albert, "Relativity", English translation by Robert Lawson, Pi Press, New York, 2005.

[2] Kennedy Robert, “A student Guide to Einstein's Major Papers", Oxford University Press, 2012.

[3] Wikipedia.com, "Special Relativity", Feb. 26, 2015.

[4] McFarland Ernie, "Einstein's Special Relativity", Trifolium Books Inc., Toronto.

[5] Schwab Adolf, "Field Theory Concepts", Springer-Verlag, New York, 1988.

[6] Wikipedia.com, “General Relativity”, Feb. 26, 2015.

[7] Everett Allen, and Thomas Roman, "Time Travel and Warp Drives", University of Chicago Press, 2012.

[8] Dahanayake Bandula, "Universe: Not Expanding", International Journal of Astrophysics and Space Science", vol. 2, No. 4, 2014, pp. 66-70. 